\title{
Fluoroquinolones and isoniazid-resistant tuberculosis: implications for the 2018 WHO guidance
}

\author{
Helen R. Stagg (1) ${ }^{1,2}$, Graham H. Bothamley (1) ${ }^{3,21}$, Jennifer A. Davidson ${ }^{4,21}$, \\ Heinke Kunst ${ }^{5,21}$, Maeve K. Lalor ${ }^{1,4,21}$, Marc C. Lipman ${ }^{6,7,21}$, \\ Miranda G. Loutet ${ }^{4,21}$, Stefan Lozewicz ${ }^{8,21}$, Tehreem Mohiyuddin ${ }^{4,21}$, \\ Aula Abbara ${ }^{9,22}$, Eliza Alexander ${ }^{10,22}$, Helen Booth ${ }^{11,22}$, Dean D. Creer ${ }^{12,22}$, \\ Ross J. Harris ${ }^{13,22}$, Onn Min Kon (10 14,22, Michael R. Loebinger ${ }^{15,22}$, \\ Timothy D. McHugh (16,22, Heather J. Milburn ${ }^{17,22}$, Paramita Palchaudhuri ${ }^{18,22}$, \\ Patrick P.J. Phillips (10 ${ }^{19,22}$, Erik Schmok ${ }^{3,22}$, Lucy Taylor ${ }^{10,22}$ and \\ Ibrahim Abubakar ${ }^{1}$, on behalf of the London INH-R TB study group ${ }^{20}$
}

@ERSpublications

WHO has assessed regimen recommendations for isoniazid-resistant TB to be of very low certainty. The addition of fluoroquinolones to a 12-month (isoniazid, rifamycin, ethambutol, short-duration pyrazinamide) regimen may be unnecessary in certain settings. http://bit.ly/2XoTgNL

Cite this article as: Stagg HR, Bothamley GH, Davidson JA, et al. Fluoroquinolones and isoniazid-resistant tuberculosis: implications for the 2018 WHO guidance. Eur Respir J 2019; 54: 1900982 [https://doi.org/ 10.1183/13993003.00982-2019].

\section{ABSTRACT}

Introduction: 2018 World Health Organization (WHO) guidelines for the treatment of isoniazid (H)-resistant ( $\mathrm{Hr}$ ) tuberculosis recommend a four-drug regimen: rifampicin (R), ethambutol (E), pyrazinamide (Z) and levofloxacin (Lfx), with or without $\mathrm{H}([\mathrm{H}] \mathrm{RZE}-\mathrm{Lfx})$. This is used once Hr is known, such that patients complete 6 months of Lfx $(\geqslant 6[\mathrm{H}] \mathrm{RZE}-6 \mathrm{Lfx})$. This cohort study assessed the impact of fluoroquinolones $(\mathrm{Fq})$ on treatment effectiveness, accounting for $\mathrm{Hr}$ mutations and degree of phenotypic resistance.

Methods: This was a retrospective cohort study of $626 \mathrm{Hr}$ tuberculosis patients notified in London, 20092013. Regimens were described and logistic regression undertaken of the association between regimen and negative regimen-specific outcomes (broadly, death due to tuberculosis, treatment failure or disease recurrence).

Results: Of 594 individuals with regimen information, 330 (55.6\%) were treated with (H)RfZE (Rf=rifamycins) and $211(35.5 \%)$ with (H)RfZE-Fq. The median overall treatment period was 11.9 months and median $\mathrm{Z}$ duration 2.1 months. In a univariable logistic regression model comparing (H)RfZE with and without Fqs, there was no difference in the odds of a negative regimen-specific outcome (baseline $(\mathrm{H})$ RfZE, cluster-specific odds ratio 1.05 (95\% CI 0.60-1.82), $\mathrm{p}=0.87$; cluster NHS trust). Results varied minimally in a multivariable model. This odds ratio dropped $(0.57,95 \%$ CI $0.14-2.28)$ when Hr genotype was included, but this analysis lacked power $(\mathrm{p}=0.42)$.

Conclusions: In a high-income setting, we found a 12-month (H)RfZE regimen with a short $\mathrm{Z}$ duration to be similarly effective for $\mathrm{Hr}$ tuberculosis with or without a Fq. This regimen may result in fewer adverse events than the WHO recommendations.

This article has supplementary material available from erj.ersjournals.com

Received: 15 May 2019 | Accepted after revision: 01 July 2019

Copyright OERS 2019. This version is distributed under the terms of the Creative Commons Attribution NonCommercial Licence 4.0. 


\section{Introduction}

Isoniazid $(\mathrm{H})$ is a key drug used in the treatment of both tuberculosis disease (TB) and latent TB infections. Research into $\mathrm{H}$-resistant $(\mathrm{Hr}) \mathrm{TB}$ has been neglected in favour of studies of simultaneous $\mathrm{Hr}$ and rifampicin (R) resistance (Rr), i.e. multidrug resistance (MDR) [1]. Globally, 7.1\% of new incident TB patients between 2003 and 2017 had $\mathrm{Hr}$ disease without associated $\mathrm{Rr}$ (henceforth known as "Hr TB"), as did 7.9\% of previously treated patients [2]. The distribution of $\mathrm{Hr}$ TB varies substantially by country $[1,3]$.

Hr has been associated with poor treatment outcomes, the need to tailor treatment regimens and the development of additional drug resistance during treatment [1]. A meta-analysis of randomised controlled trial (RCT) data, controlling for regimen, demonstrated that incidence rates of treatment failure were 10.9 times higher in $\mathrm{Hr} \mathrm{TB}$ versus drug-sensitive disease (95\% CI 5.9-20) [4]. In the same study, relapse rates in $\mathrm{Hr} \mathrm{TB}$ were 1.8-fold higher (1.2-2.6) and acquired drug resistance 5.1 times higher (2.3-11.0).

Given these concerns, policymakers have issued specific treatment guidance for Hr TB. In 2018 the World Health Organization (WHO) conditionally recommended a regimen of $\mathrm{R}$, ethambutol (E), pyrazinamide (Z) and levofloxacin (Lfx) with or without $\mathrm{H}$ ([H]RZE-Lfx), to be initiated once Hr is confirmed [5]. If treatment starts before $\mathrm{Hr}$ is known, it is continued until Lfx is used for 6 months, even if the duration of the other drugs is therefore longer $(\geqslant 6[\mathrm{H}]$ RZE-6Lfx $)$. In the absence of rapid molecular testing for $\mathrm{Hr}$, overall treatment duration is thus 7.5-9 months, depending upon whether liquid or solid culture is used [6]. WHO has assessed the evidence underlying this regimen to be of very low certainty [5]. Within the UK, the National Institute for Health and Care Excellence recommends a 9-month regimen of 2 months of RZE, followed by 7 months of RE [7]. This can be extended to 12 months' duration (10-month continuation phase), if disease is extensive. The American Thoracic Society is currently revising its guidance [8]. In 2003, they recommended a 6-month regimen of RZE, plus a fluoroquinolone (Fq) for extensive disease [9]. All bodies acknowledge the need for future studies to optimise regimens, e.g. to determine the implications of the resistance-causing $\mathrm{Hr}$ mutation(s).

In light of the 2018 WHO recommendations, we undertook a retrospective cohort study to identify the treatment regimens currently being used for $\mathrm{Hr} \mathrm{TB}$ in a high-income setting with universal healthcare (London, UK). We assessed the importance of including Fqs during treatment, accounting for baseline Hr phenotype and genotype.

\section{Methods}

\section{Study population}

We included all patients aged $\geqslant 18$ years notified in England (as a statutory requirement) to Public Health England (PHE)'s Enhanced TB Surveillance system (ETS) between January 1, 2009 and December 31, 2013 with disease caused by phenotypically $\mathrm{Hr}$ Mycobacterium tuberculosis. Baseline demographic and basic clinical and microbiological data were available from PHE. Individuals notified in London formed the retrospective cohort; additional data collection for these individuals is described below.

\section{Treatment regimens}

Detailed regimen, adherence and regimen-specific outcome information was gathered from clinical notes at the last hospital to treat the patient recorded by PHE (supplementary file 1).

Affiliations: ${ }^{1}$ Institute for Global Health, University College London, London, UK. ${ }^{2}$ Usher Institute of Population Health Sciences and Informatics, University of Edinburgh, Edinburgh, UK. ${ }^{3}$ Respiratory Medicine, Homerton University Hospital, London, UK. ${ }^{4}$ Tuberculosis Unit, National Infection Service, Public Health England, London, UK. ${ }^{5}$ Blizard Institute, Barts and The London School of Medicine and Dentistry, Queen Mary University of London, London, UK. ${ }^{6}$ Respiratory Medicine, Royal Free Hospital, London, UK. ${ }^{7}$ UCL Respiratory, Division of Medicine, University College London. ${ }^{8}$ Respiratory Medicine, North Middlesex University Hospital, London, UK. ${ }^{9}$ Infectious Diseases, London North West University Healthcare NHS Trust, London, UK.

${ }^{10}$ National Mycobacterial Reference Service South, Public Health England, London, UK. ${ }^{11}$ Tuberculosis Service, University College London Hospitals/Whittington Health, London, UK. ${ }^{12}$ Respiratory Medicine, Barnet General Hospital, Royal Free London NHS Foundation Trust, London, UK. ${ }^{13}$ Statistics, Modelling and Economics Department, Public Health England, London, UK. ${ }^{14}$ TB Service, Imperial College Healthcare, London, UK. ${ }^{15}$ Respiratory Medicine, Chelsea and Westminster Hospital, London, UK. ${ }^{16}$ Centre for Clinical Microbiology, University College London, London, UK. ${ }^{17}$ Respiratory Medicine, Guy's and St Thomas' Hospital, London, UK. ${ }^{18}$ Respiratory Services, Queen Elizabeth Hospital, London, UK. ${ }^{19}$ Dept of Medicine and Dept of Epidemiology and Biostatistics, University of California San Francisco, San Francisco, CA, USA. ${ }^{20}$ Additional London INH-R TB study group members are listed in the acknowledgements section. ${ }^{21}$ These authors contributed equally to this manuscript and are presented alphabetically. ${ }^{22}$ These authors contributed equally to this manuscript and are presented alphabetically.

Correspondence: Helen R. Stagg, Usher Institute of Population Health Sciences and Informatics, University of Edinburgh, Edinburgh, EH8 9DX, UK. E-mail: helen.staggded.ac.uk 
Regimens were described and categorised. The rifamycins (Rf) R and rifabutin were grouped together, as were the injectables, Fqs other than moxifloxacin $(\mathrm{M})$, and the previously named group 4/5 drugs [10, 11]. A binary regimen variable was created of RfZE regimens in the presence or absence of $\mathrm{H}$ with or without an additional Fq: (H)RZE versus (H)RZE-Fq/M. If additional drugs were included, the regimen was not counted within the binary variable.

The presence of high-dose $\mathrm{H}$ within the regimen was documented, as was whether $\mathrm{Rf}, \mathrm{Z}$ or $\mathrm{E}$ were dosed thrice weekly (as opposed to more frequently). The length of time a patient was treated before the regimen was adapted to account for $\mathrm{Hr}$ (which was dependent on the duration of drug sensitivity testing (DST)) was grouped 0 to $<2,2$ to $<6$ and $\geqslant 6$ months.

\section{Genotyping and phenotyping}

Phenotypic DSTs for first-line drugs were conducted on baseline samples. DSTs for second-line drugs were conducted if resistance to $\mathrm{R}$ or two or more other first-line drugs (but not $\mathrm{H}$ alone, although this could be requested) was detected. These results were recorded within ETS. Patients were grouped according to the baseline drug resistance pattern of their disease.

The degree of phenotypic resistance to $\mathrm{H}$ was extracted from the National Mycobacterium Reference Service (NMRS)-South system (supplementary file 1).

Whole-genome sequencing (WGS) to detect resistance mutations was undertaken for a subset of patients among those notified 2012-2013 using an Illumina HiSeq (San Diego, CA, USA) at the PHE central sequencing unit [12].

\section{Other exposure variables}

Age, sex, being born in the UK, ethnic group, social risk factors (homelessness, problematic drug use, problematic alcohol use and imprisonment), previous diagnosis of $\mathrm{TB}$ and inpatient information came from ETS. Decisions surrounding the grouping of these variables are documented in supplementary file 1.

An outbreak of Hr TB has been present in (mainly north) London since 1995 [13, 14]. Due to awareness of this outbreak among clinicians, patients with epidemiological risk factors consistent with the outbreak (in which nonadherence was common and treatment outcomes poor) may have been treated differently from other patients.

An additional variable documented if a patient had issues adhering to treatment, according to their clinical notes (supplementary file 1).

\section{Outcomes}

A patient's treatment period for $\mathrm{Hr} \mathrm{TB}$ is made up of up to three components: the regimen used prior to $\mathrm{Hr}$ being known; the regimen used once $\mathrm{Hr}$ is known; and (potentially) a further regimen or regimens if the $\mathrm{Hr}$ regimen is insufficiently effective. Overall treatment outcomes (available in ETS) capture this entire period. Regimen-specific outcomes, taken from clinical notes, document the effectiveness of the $\mathrm{Hr}$ regimen and thus capture only the first two components (table 1). For the regression model, the neutral and positive groups were merged to create a binary outcome.

\section{Analysis}

Data were cleaned in Microsoft Excel (Redmond, WA, USA) and analysed in Stata 15 (StataCorp, College Station, TX, USA).

The characteristics of the London cohort were assessed. Descriptive analyses of the regimens used were undertaken, followed by regression analyses. Initially, individuals with additional phenotypic drug resistance identified in baseline samples taken were excluded from the regression models, unless resistance was to streptomycin (S). This was because $S$ is not routinely used in the treatment of drug-sensitive or MDR TB in the UK $[7,16]$. Random-effects univariable logistic regression models were built to examine the impact of different factors on the likelihood of negative regimen-specific outcomes, with a random effect included on National Health Service (NHS) trust to adjust for clustering.

A multivariable logistic regression model was then built, using the binary regimen categorisation as the main exposure and including a random effect on NHS trust. Details of confounder selection, etc. are presented in supplementary file 1 .

\section{Sensitivity and extended analyses}

Four additional logistic regression models were run. The first included Hr genotyping results. Next, adherence was substituted for thrice-weekly dosing. The third included all patients, regardless of whether 
TABLE 1 Classification of regimen-specific outcomes

Components

Negative Treatment completed, followed by recurrence; outcome missing, but recurrence; or neutral outcome, followed by recurrence

Died due to TB or TB-associated death $\geqslant 2$ weeks after starting treatment

Treatment stopped early or regimen changed due to worsening/ not improving, treatment failure, adverse events or the development of additional drug resistance

Additional drug resistance developed during treatment

Neutral Died from TB or TB-associated death within 2 weeks of starting treatment

Died from non-TB related causes, or cause unknown

Treatment stopped early or regimen changed due to non-adherence, loss to follow-up, patient choosing to cease their medication, pregnancy or comorbidities

Patient transferred to another hospital during their treatment

Positive Treatment completed as initially prescribed (once Hr known); or treatment completed, no recurrence

\section{Comments}

Recurrence of disease $\geqslant 12$ months after notification; recurrences documented until the end of 2015 (the most recent available data at the time of analysis); if disease recurred after the end of treatment at any time and the patient re-presented to the same hospital, this was classified as a negative outcome

Death before 2-week threshold considered to be too early to be influenced by the treatment [15]

Length extended, antibiotics added/removed, frequency altered, dose altered, treatment stopped

To any drug

Death before 2-week threshold considered to be too early to be influenced by the treatment [15]

No further documentation; transfer before any negative outcomes occurred

Regimen-specific outcomes (extracted from clinical notes) presented in detail. The first outcome arising per patient was documented, unless a negative outcome occurred after one that is neutral. TB: tuberculosis; Hr: isoniazid resistance.

they were resistant to drugs in addition to $\mathrm{H}$ (and S). The fourth was a post hoc model adjusting for factors associated with the use of Fqs.

\section{Ethical permissions}

PHE is legislated by the National Information Governance Board for Health and Social Care to hold and analyse surveillance data for public health purposes under section 251 of the NHS Act 2006. This retrospective cohort study was approved by the London Camberwell St Giles research ethics committee (16/LO/1269) and, in addition, given permission to undertake data extraction without consent under section 251 (Confidentiality Advisory Group reference 16/CAG/0092).

FIGURE 1 Flow chart of participants. $\mathrm{Hr}$ : isoniazid-resistant; TB: tuberculosis; PHE: Public Health England. n=1228 Hr TB patients notified to PHE 2009-2013

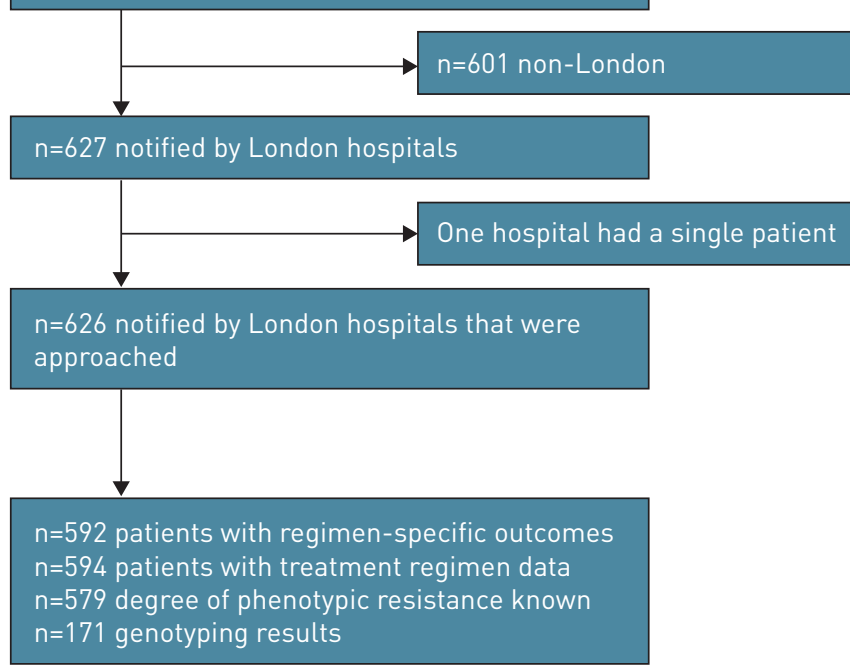


TABLE 2 Demographic and clinical baseline characteristics of the 626 individuals in the London cohort

\begin{tabular}{|c|c|}
\hline Overall & $626(100)$ \\
\hline \multicolumn{2}{|l|}{ Year } \\
\hline 2009 & 137 (21.9) \\
\hline 2010 & 118 (18.8) \\
\hline 2011 & $141(22.5)$ \\
\hline 2012 & $125(20.0)$ \\
\hline 2013 & 105 (16.8) \\
\hline Missing & $0(0.0)$ \\
\hline \multicolumn{2}{|l|}{ Sex } \\
\hline Male & $380(60.7)$ \\
\hline Female & 246 (39.3) \\
\hline Missing & $0(0.0)$ \\
\hline \multicolumn{2}{|l|}{ Age years } \\
\hline $18-37$ & $358(57.2)$ \\
\hline $38-57$ & 199 (31.8) \\
\hline $58-77$ & $62(9.9)$ \\
\hline$\geqslant 78$ & $7(1.1)$ \\
\hline Missing & $0(0.0)$ \\
\hline \multicolumn{2}{|l|}{ UK born } \\
\hline No & 497 (79.4) \\
\hline Yes & 121 (19.3) \\
\hline Missing & $8(1.3)$ \\
\hline \multicolumn{2}{|l|}{ Ethnic group } \\
\hline White & $97(15.5)$ \\
\hline Black African & 125 (20.0) \\
\hline Black Other & $45(7.2)$ \\
\hline Indian subcontinent & $270(43.1)$ \\
\hline Other & $85(13.6)$ \\
\hline Missing & $4(0.6)$ \\
\hline \multicolumn{2}{|l|}{ Social risk factors } \\
\hline No or unknown & 510 (81.5) \\
\hline One or more ever & $37(5.9)$ \\
\hline One or more current & $79(12.6)$ \\
\hline \multicolumn{2}{|l|}{ Previous TB diagnosis } \\
\hline No & 575 (91.9) \\
\hline Yes & $20(3.2)$ \\
\hline Missing & $31(5.0)$ \\
\hline \multicolumn{2}{|l|}{ Inpatient } \\
\hline No & $422(67.4)$ \\
\hline Yes & $190(30.4)$ \\
\hline Missing & $14(2.2)$ \\
\hline \multicolumn{2}{|l|}{ Site of disease } \\
\hline Pulmonary \pm extrapulmonary, smear +ve & 194 (31.0) \\
\hline Pulmonary \pm extrapulmonary, smear -ve & $159(25.4)$ \\
\hline Meningeal TB or other CNS involvement & $24(3.8)$ \\
\hline Other extrapulmonary & 249 (39.8) \\
\hline Missing & $0(0.0)$ \\
\hline \multicolumn{2}{|l|}{ Part of outbreak } \\
\hline No & $501(80.0)$ \\
\hline Yes & 65 (10.4) \\
\hline Missing & $60(9.6)$ \\
\hline \multicolumn{2}{|l|}{ Any additional drug resistance } \\
\hline No & $453(72.4)$ \\
\hline Yes & $173(27.6)$ \\
\hline
\end{tabular}

Data are presented as $\mathrm{n}$ (column \%). TB: tuberculosis; CNS: central nervous system; \pm : with or without; -ve: negative; +ve: positive.

\section{Results}

Patient population

1228 individuals with $\mathrm{Hr}$ TB were notified in England between 2009 and 2013 (figure 1). Of these, $626(51.0 \%)$ were notified by 31 hospitals (supplementary file 2 ) in London (19 NHS trusts). One hospital 
TABLE 3 Regimen-specific outcomes and availability of regimen data

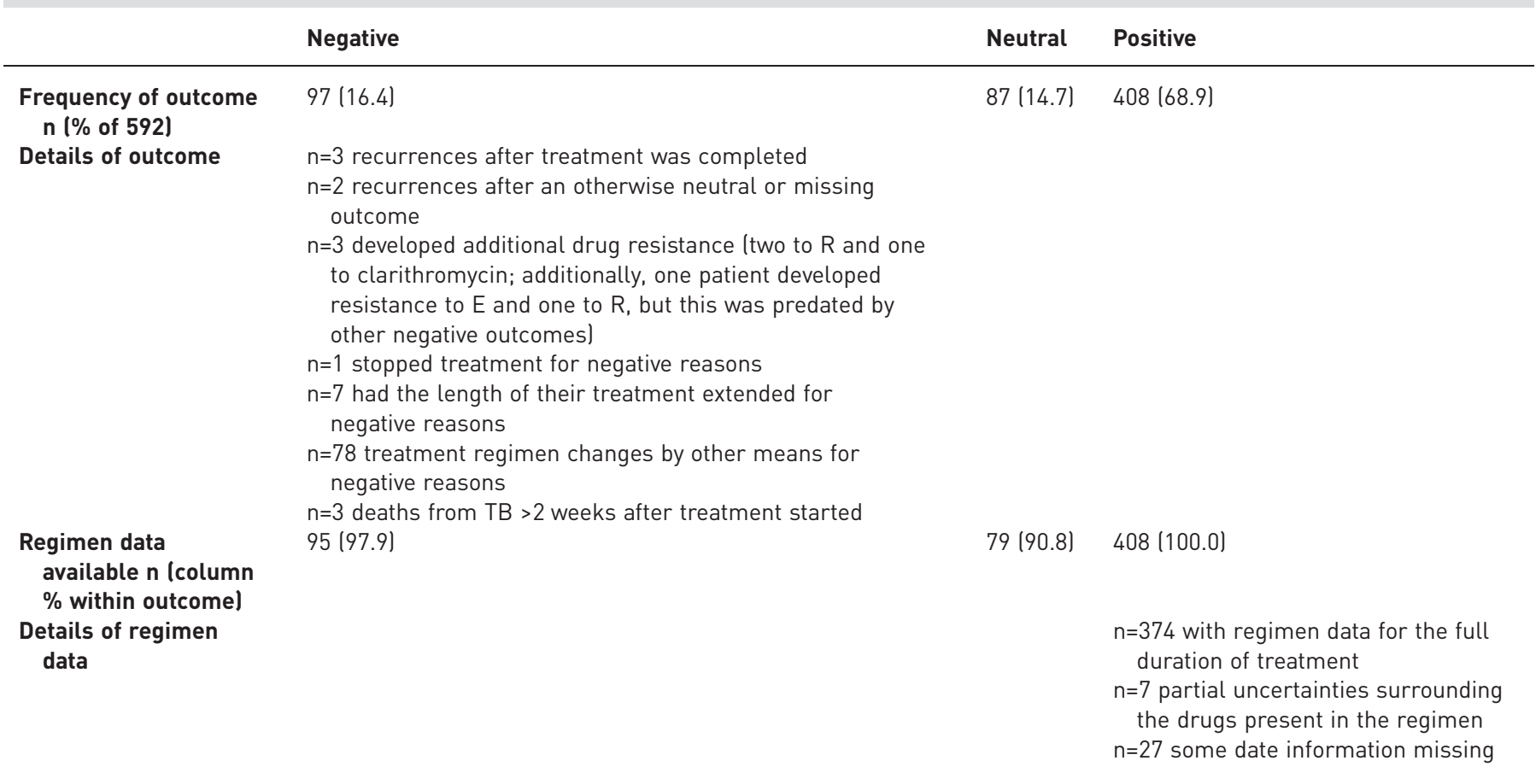

Regimen-specific outcomes and treatment regimen availability for the 592 (94.6\%) out of 626 of individuals with an outcome recorded in the London cohort. E: ethambutol; R: rifampicin; TB: tuberculosis.

had only a single patient and was not approached for local approvals. The baseline characteristics of the London cohort are described in table 2.

Phenotypic testing for non-H drug resistance revealed that 173 (27.6\%) out of 626 patients within the London cohort had additional drug resistance at baseline (supplementary file 3). The most common resistance was towards S (139 (22.2\%) out of 626).

The majority of samples were documented in the NMRS system as highly $\mathrm{Hr}$ at baseline (495 (79.1\%) out of 626). Three (0.5\%) displayed borderline results; one was listed as drug sensitive $(0.2 \%)$; and $35(5.6 \%)$ were present in the system, but did not have their Hr levels logged. 47 individuals could not be found within NMRS, but were recorded as $\mathrm{Hr}$ within ETS.

\section{Regimen-specific outcomes}

Regimen-specific outcomes were available for 592 (94.6\%) out of 626 patients (table 3). 97 (16.4\%) had a negative outcome.

Relationship between treatment regimens and regimen-specific outcomes

Of the 626 patients, $582(93.0 \%)$ had both a regimen-specific outcome recorded and treatment information. Of these, 538 (92.4\%) were not resistant to drugs in addition to $\mathrm{H}$, apart from $\mathrm{S}$, and 84 had a negative regimen-specific outcome (three of which were recurrences). 498 (92.6\%) out of 538 were treated with $(\mathrm{H}) \mathrm{RfZE}$ or $(\mathrm{H}) \mathrm{RfZE}-\mathrm{Fq} / \mathrm{M}$ (table 4). For a more detailed description of the treatment regimens, see supplementary file 4 .

Differences in the odds of a negative regimen-specific outcome were not detected between patients treated with (H)RfZE (baseline) and (H)RfZE-Fq/M (cluster-specific OR 1.02, 95\% CI 0.59-1.77; p=0.93; table 4). None of the other treatment regimens or associated factors were found to be associated with the odds of negative outcomes (table 4, supplementary file 5). We observed more negative outcomes with the use of thrice weekly dosing versus more frequent dosing (OR 1.81, 95\% CI 0.83-3.94), but this may have been a chance finding $(\mathrm{p}=0.15)$.

Seven exposure variables/confounders were included in the multivariable model: regimen, thrice-weekly dosing, Hr phenotype, sex, age (linear variable), ethnic group and previous TB diagnosis. Evidence for effect modification was not found. In the final multivariable model of 435 patients (table 5), there was no 


\begin{tabular}{|c|c|c|c|c|}
\hline & $\begin{array}{l}\text { Overall n } \\
\text { (column \%) }\end{array}$ & $\begin{array}{c}\text { Negative outcome } \\
\text { n (row \%) }\end{array}$ & OR $(95 \% \mathrm{CI})$ & p-value \\
\hline Overall & 538 (100) & $84(15.6)$ & & \\
\hline \multicolumn{5}{|l|}{ Regimen } \\
\hline (H)RfZE & $306(56.9)$ & $46(15.0)$ & & 0.93 \\
\hline (H)RfZE-Fq/M & $192(35.7)$ & $30(15.6)$ & $1.02(0.59-1.77)$ & \\
\hline Missing & $40(7.4)$ & $8(20.0)$ & & \\
\hline \multicolumn{5}{|l|}{ Thrice-weekly dosing } \\
\hline More frequent & $464(86.2)$ & $66(14.2)$ & & 0.15 \\
\hline Thrice weekly & 53 (9.9) & $12(22.6)$ & $1.81(0.83-3.94)$ & \\
\hline Missing & $21(3.9)$ & $6(28.6)$ & & \\
\hline \multicolumn{5}{|l|}{ Time before Hr known } \\
\hline 0 to $<2$ months & $325(60.4)$ & $56(17.2)$ & & 0.27 \\
\hline 2 to $<6$ months & $159(29.6)$ & $18(11.3)$ & $0.62(0.34-1.13)$ & \\
\hline$\geqslant 6$ months & $10(1.9)$ & $2(20.0)$ & $1.11(0.22-5.66)$ & \\
\hline Missing & $44(8.2)$ & $8(18.2)$ & & \\
\hline \multicolumn{5}{|l|}{ Phenotype } \\
\hline Highly resistant & 442 (82.2) & $69(15.6)$ & & 0.73 \\
\hline Resistant & $36(6.7)$ & $5(13.9)$ & $0.88(0.32-2.39)$ & \\
\hline Borderline, sensitive or results not logged & $29(5.4)$ & $6(20.7)$ & $1.46(0.55-3.88)$ & \\
\hline Missing & $31(5.8)$ & $4(12.9)$ & & \\
\hline \multicolumn{5}{|l|}{ Adherence issues or treatment gaps } \\
\hline No or unknown & $425(79.0)$ & $64(15.1)$ & & 0.29 \\
\hline Not severe or of unknown severity & $56(10.4)$ & $13(23.2)$ & $1.62(0.80-3.28)$ & \\
\hline Severe & $57(10.6)$ & $7(12.3)$ & $0.72(0.30-1.73)$ & \\
\hline
\end{tabular}

Univariable logistic regression of treatment regimen and associated factors as predictors of negative regimen-specific outcomes. Included patients were notified in London, had regimen-specific outcome and regimen information, and their disease was without additional drug resistance, unless to streptomycin. Each model contains the patients without missing data. H: isoniazid; Rf: rifamycin; Z: pyrazinamide; E: ethambutol; Fq: fluoroquinolones; M: moxifloxacin; Hr: isoniazid resistance.

discernible difference in the odds of a negative outcome between the two regimens (OR 0.99, 95\% CI 0.53-1.85; $\mathrm{p}=0.97$ ). The association between thrice-weekly dosing and negative outcomes was slightly strengthened in terms of the effect estimate (OR 2.34, 95\% CI (0.90-6.09), although the association observed could still have been due to chance $(\mathrm{p}=0.09)$.

\section{Impact of genotype and other sensitivity analyses}

The most common $\mathrm{Hr}$ genotypes observed were fabG1 C-15 T (87 (50.9\%) out of 171) and katG S315 T (75 (43.9\%) out of 171; supplementary file 6). For 10 (5.8\%) out of 171 strains, sequencing either failed, no resistance mutations were detected or it was not known whether the single nucleotide polymorphisms (SNPs) found generate drug resistance.

In a univariable model, no difference was seen in the likelihood of a negative treatment outcome between the $k a t G \mathrm{~S} 315 \mathrm{~T} / \mathrm{N}$ genotypes and a fabG1 C-15 T baseline (OR 1.17, 95\% CI 0.42-3.31; p=0.76). In a multivariable model, evidence for effect modification by genotype was not detected. Genotype was not independently associated with the outcome (supplementary file 7). In this model, there was a suggestion that the odds of a negative regimen-specific outcome were reduced for (H)RfZE-Fq/M versus (H)RfZE (OR 0.57, 95\% CI 0.14-2.28), but we were underpowered for this analysis $(\mathrm{p}=0.42)$.

Inclusion of other potential confounder sets in the multivariable model did not impact our findings (supplementary file 8).

\section{Discussion}

In this analysis of $\mathrm{Hr} \mathrm{TB}$ patients notified by London hospitals between 2009 and 2013, 16.4\% of individuals had a negative outcome. (H)RfZE and (H)RfZE-Fq/M regimens were taken by $92.6 \%$ of individuals without additional drug resistance (apart from to $\mathrm{S}$ ) and with both regimen and regimen-specific outcome data. Among these patients, we found no discernible difference in the odds of a negative regimen-specific outcome between $(\mathrm{H}) \mathrm{RfZE}$ and $(\mathrm{H}) \mathrm{RfZE}-\mathrm{Fq} / \mathrm{M}$ regimens. Examining individuals 


\begin{tabular}{|c|c|c|}
\hline & OR $(95 \% \mathrm{CI})$ & p-value \\
\hline \multicolumn{3}{|l|}{ Regimen } \\
\hline (H)RfZE & & 0.97 \\
\hline (H)RfZE-Fq/M & $0.99(0.53-1.85)$ & \\
\hline \multicolumn{3}{|l|}{ Thrice-weekly dosing } \\
\hline More frequent & & 0.09 \\
\hline Thrice weekly & 2.34 (0.90-6.09) & \\
\hline \multicolumn{3}{|l|}{ Phenotype } \\
\hline Highly resistant & & 0.66 \\
\hline Resistant & $0.64(0.17-2.43)$ & \\
\hline $\begin{array}{l}\text { Borderline, sensitive or results not logged } \\
\text { Missing }\end{array}$ & $1.40(0.45-4.31)$ & \\
\hline \multicolumn{3}{|l|}{ Sex } \\
\hline Male & & 0.02 \\
\hline Female & $2.05(1.13-3.71)$ & \\
\hline \multicolumn{3}{|l|}{ Age years } \\
\hline $18-37$ & & 0.46 \\
\hline Per 20-year increase & $1.18(0.75-1.86)$ & \\
\hline \multicolumn{3}{|l|}{ Ethnic group } \\
\hline White & & 0.15 \\
\hline Black African & $0.42(0.15-1.18)$ & \\
\hline Black Other & $0.33(0.08-1.39)$ & \\
\hline Indian subcontinent & $0.58(0.23-1.45)$ & \\
\hline Other & $1.10(0.42-2.92)$ & \\
\hline \multicolumn{3}{|l|}{ Previous TB diagnosis } \\
\hline No & & 0.13 \\
\hline Yes & $3.12(0.75-12.91)$ & \\
\hline
\end{tabular}

Multivariable logistic regression of treatment regimen as a predictor of negative regimen-specific outcomes in patients without additional drug resistance, unless to streptomycin, adjusted for all variables in the table. Model contains 435 patients. H: isoniazid; Rf: rifamycin; Z: pyrazinamide; E: ethambutol; Fq: fluoroquinolones; M: moxifloxacin; TB: tuberculosis.

with a positive treatment outcome, the overall duration of treatment was generally 12 months, with $\mathrm{Z}$ durations of 2 months in the initiation phase. After adjustment for $\mathrm{Hr}$ genotype, the likelihood of a negative outcome was found to be lower among individuals treated with (H)RfZE-Fq/M, but this analysis was underpowered.

Our findings sit in the context of preceding work on the relative efficacy and effectiveness of different regimens for $\mathrm{Hr} \mathrm{TB}$, including four meta-analyses [17-20]. FREGONESE et al.'s [17] individual-level patient meta-analysis, the foundation of the 2018 WHO guidelines, showed a value for including a $\mathrm{Fq}$ in continuous (H)RZE regimens, and suggested equivalence between 6 and 8-9 months of (H)RZE. The WHO acknowledges that overall treatment length findings may be subject to confounding by indication, due to patients with more complex sites of disease receiving longer regimens [5].

Notably, global RCT evidence for the effectiveness of Fqs in non-MDR-TB derive solely from the Rifaquin trial, as ReMox did not demonstrate non-inferiority when $H$ was replaced with $M$ for non-MDR TB [21, 22]. When considering the choice of $\mathrm{Fq}$, although the WHO recommends the use of Lfx, $\mathrm{M}$ was generally used in our study. Within FREGONESE et al. [17], roughly equal numbers of studies used these two drugs, which were not directly compared. However, comparative data are available from a MDR-TB trial (no difference in treatment outcomes when comparing the two drugs; fewer adverse events for $\mathrm{M}$ ) [23], and rabbit and mouse models (M broadly superior over Lfx) [24, 25]. Lfx doses in such studies may have been too low [26, 27]. Further RCTs are required.

The above meta-analyses were unable to thoroughly consider the role of Hr genotype and phenotype in treatment decisions; the evidence from previous observational studies is unclear $[1,28]$. Where adjustment for genotype in observational studies has been undertaken, it was largely for inhA and kat $G$. In our cohort, with a very high prevalence of $f a b G 1$ in addition to $k a t G$ mutations, we find an indication that the $\mathrm{Hr}$ genotype is influential. fabG1 is part of the inhA operon and is involved in fatty acid synthesis; SNPs within the gene are known to confer $\operatorname{Hr}[29,30]$. 
The evidence currently underpinning global treatment guidelines for $\mathrm{Hr} \mathrm{TB}$ is limited. Our study adds to this discussion, including consideration of the effect of resistance phenotype and genotype on the regimen-outcomes relationship. Importantly, in our core analysis, 192 patients received a $\mathrm{Fq}$ in addition to (H)RfZE, which provides substantial new evidence to that presented by FrEGONESE et al. [17], whose analysis of treatment success included 251 patients receiving a Fq. Our findings did not differ when site of disease was adjusted for as a confounder (including meningeal TB or other central nervous system involvement; data not shown) and when patients with additional drug resistance were included.

Within this study, actual rather than intended treatment durations were captured, which prevented us from undertaking analyses of the impact of overall or drug-specific durations. Importantly, however, when considering 9 versus 12 months of treatment, the majority of negative outcomes occurred before 9 months and the number of relapses was small, with two of the three occurring after $>15$ months of treatment. Thus our data may indicate the potential to shorten treatment to 9 months in our setting. Some patient notes could not be accessed as patients had died. This was unlikely to have been of a magnitude sufficient to bias our findings. We did not differentiate between recurrence due to relapse versus reinfection, and thus may have overestimated the number of negative outcomes (nondifferential misclassification). Gaps in phenotypic data arose due to 1) missing records within NMRS from a specific period and reference laboratory; and 2) incomplete data entry into NMRS from the reference laboratory (cross-tabulations against patient characteristics did not indicate that this particularly affected any specific patient groups). The phenotypic and genotypic $\mathrm{Hr}$ patterns documented summarise that of the overall bacterial population; the presence of minor strains will not have been captured. Our findings about thrice-weekly dosing may represent the use of such a dosing pattern specifically among patients where directed observation of treatment was deemed necessary. HIV status, a potential confounder, was not obtainable during data collection.

Despite these limitations, there are important ramifications for our findings both nationally and internationally. We document a drug combination that differs from that recommended (with very low certainty) by the WHO [5], which may be as effective. We note that, if the overall duration of treatment is long enough (12 months), a Fq may not be necessary in certain settings, even with relatively short durations (median 2 months in the initiation phase) of Z. Notably, in settings where DST occurs via phenotyping from cultures, the WHO regimen $\geqslant 6[\mathrm{H}] \mathrm{RZE}-6 \mathrm{Lfx}$ is likely to have total duration of 7.5-9 months, when time to result is considered. This also affects the longer regimen in their 6- versus 8-9-month duration comparison; the latter translates to 9.5-12 months. By comparison, in settings undertaking rapid genotyping directly from patient samples, the WHO regimen duration would be 6 months and the average duration documented here $\sim 10$ months.

Global regimen choices will depend upon the trade-off between patient desire for regimens of minimal length, adherence concerns, adverse events, ease of administration and cost. Costs are raised if fixed dose combination pills cannot be used and Fqs are added in. When it comes to comparing the likelihood of adverse events, the trade-off would be between a longer duration of $\mathrm{E}$, but shorter duration of $\mathrm{Z}$ in our predominantly used regimen, versus continued $\mathrm{Z}$ and the addition of LfX, as per the WHO recommendations. Each of these drugs has its own distinct adverse event profile [23, 31].

Fq DST results are important when deciding on Fq use within a $\mathrm{Hr}$ regimen. Only 48 individuals in the London cohort had their baseline samples tested for resistance to M. In 2018, PHE rolled-out prospective WGS to provide routine resistance predictions and mutation identification, thus improving the rapidity of DST and coverage of second-line testing. New molecular Hr tests can also aid rapidity, as the use of WGS still depends on culture [32].

Within the limitations of an observational study, where the use of Fqs was not randomised, we find in a high-income setting with comprehensive patient management, a 12-month (H)RfZE regimen with a short $\mathrm{Z}$ duration to be similarly effective for $\mathrm{Hr} \mathrm{TB}$, with or without a Fq. Hr genotype may influence these findings. In the absence of Fqs and long durations of $\mathrm{Z}$, this regimen may have fewer adverse events than the WHO recommended $\geqslant 6[\mathrm{H}] \mathrm{RZE}-6 \mathrm{Lfx}$. RCTs should be undertaken to provide stronger global recommendations.

Acknowledgements: H.R. Stagg would also like to acknowledge the National Health Service and Research and Development office staff at different hospital sites, who are too numerous to name individually, but without whom this study would not have been possible. The authors also wish to thank Patryk Solinski, a Patient and Public Involvement representative on H.R. Stagg's Fellowship Advisory Panel and to acknowledge the following clinicians for their help with data collection: David Adeboyeku (Central Middlesex Hospital) and Devan Vaghela (Barts Health NHS Trust).

Additional London INH-R TB study group members are as follows (please note that these individuals contributed equally and are presented in alphabetical order): Lucy V. Baker, Respiratory Medicine, University Hospital Lewisham, London, UK; Jessica C. Barrett, Infectious Diseases, London North West University Healthcare NHS Trust, London, UK; Helen Burgess, Respiratory Medicine, West Middlesex University Hospital, London, UK; Catherine Cosgrove, 
Infectious Diseases, St George's Hospital, London, UK; Anne Dunleavy, Respiratory Medicine, St George's Hospital, London, UK; Marie Francis, Institute for Global Health, University College London, London, UK; Urmi Gupta, Respiratory Medicine, King George Hospital, London, UK; Shahid Hamid, Respiratory Medicine, Princess Royal University Hospital, London, UK; Brigitte M. Haselden, Respiratory Medicine, The Hillingdon Hospital NHS Foundation Trust, London, UK; Emma Holden, Respiratory Medicine, Kingston Hospital, London, UK; Vanessa Kahr Respiratory Services, St Helier Hospital, London, UK; William Lynn, Infectious Diseases, London North West University Healthcare NHS Trust, London, UK; Felicity M. Perrin, Respiratory Medicine, King's College Hospital NHS Foundation Trust, London, UK; Ananna Rahman, Respiratory Medicine Service, Barts Health NHS Trust, London, UK; and Mohammad R. Soobratty, Respiratory Services, Croydon University Hospital, London, UK.

Support statement: This report is independent research supported by the National Institute for Health Research (Post Doctoral Fellowship, Helen R. Stagg, PDF-2014-07-008). The views expressed in this publication are those of the author (s) and not necessarily those of the NHS, the National Institute for Health Research or the Department of Health. The sponsors had no role in the study design; collection, analysis and interpretation of the data; writing of the report; and in the decision to submit the paper for publication. Funding information for this article has been deposited with the Crossref Funder Registry.

Conflict of interest: H.R. Stagg reports grants from National Institute for Health Research, UK (PDF-2014-07-008), during the conduct of the study; grants from Medical Research Council, UK (MC_PC_17101) and Korea Health Industry Development Institute, outside the submitted work. G.H. Bothamley has nothing to disclose. J.A. Davidson has nothing to disclose. H. Kunst has nothing to disclose. M.K. Lalor has nothing to disclose. M.C. Lipman has nothing to disclose. M.G. Loutet has nothing to disclose. S. Lozewicz has nothing to disclose. T. Mohiyuddin has nothing to disclose. A. Abbara has nothing to disclose. E. Alexander reports personal fees for advisory board work from Insmed, outside the submitted work. H. Booth has nothing to disclose. D.D. Creer has nothing to disclose. R.J. Harris has nothing to disclose. O.M. Kon has nothing to disclose. M.R. Loebinger has nothing to disclose. T.D. McHugh has nothing to disclose. H.J. Milburn has nothing to disclose. P. Palchaudhuri has nothing to disclose. P.P.J. Phillips has nothing to disclose. E. Schmok has nothing to disclose. L. Taylor has nothing to disclose. I. Abubakar reports grants from NIHR and MRC, outside the submitted work.

\section{References}

1 Stagg HR, Lipman MC, McHugh TD, et al. Isoniazid-resistant tuberculosis: a cause for concern? Int J Tuberc Lung Dis 2017; 21: 129-139.

2 World Health Organization (WHO). Global Tuberculosis Report 2018. Geneva, WHO. www.who.int/tb/ publications/global_report/en/ Date last updated: September 2018. Date last accessed: April 2018.

3 Jenkins HE, Zignol M, Cohen T. Quantifying the burden and trends of isoniazid resistant tuberculosis, 1994-2009. PLoS One 2011; 6: e22927.

4 Menzies D, Benedetti A, Paydar A, et al. Effect of duration and intermittency of rifampin on tuberculosis treatment outcomes: a systematic review and meta-analysis. PLoS Med 2009; 6: e1000146.

$5 \quad$ World Health Organization (WHO). WHO Treatment Guidelines For Isoniazid-Resistant Tuberculosis: Supplement to the WHO Treatment Guidelines for Drug-Resistant Tuberculosis. Geneva, WHO. www.who.int/tb/publications/ 2018/WHO_guidelines_isoniazid_resistant_TB/en/ Date last updated: July 2018. Date last accessed: July 2018.

6 Drobniewski F, Cooke M, Jordan J, et al. Systematic review, meta-analysis and economic modelling of molecular diagnostic tests for antibiotic resistance in tuberculosis. Health Technol Assess 2015; 19: 1-188.

7 National Institute for Health and Care Excellence. Tuberculosis. www.nice.org.uk/guidance/ng33/resources/ tuberculosis-prevention-diagnosis-management-and-service-organisation-1837390683589 Date last updated: January 2016. Date last accessed: April 2018.

8 Nahid P, Dorman SE, Alipanah N, et al. Official American Thoracic Society/Centers for Disease Control and Prevention/Infectious Diseases Society of America Clinical Practice Guidelines: Treatment of Drug-Susceptible Tuberculosis. Clin Infect Dis 2016; 63: e147-e195.

9 Blumberg HM, Burman WJ, Chaisson RE, et al. American Thoracic Society/Centers for Disease Control and Prevention/Infectious Diseases Society of America: treatment of tuberculosis. Am J Respir Crit Care Med 2003; 167: 603-662.

10 Tiberi S, Scardigli A, Centis R, et al. Classifying new anti-tuberculosis drugs: rationale and future perspectives. Int J Infect Dis 2017; 56: 181-184.

11 World Health Organization (WHO). WHOTreatment Guidelines for Drug-Resistant Tuberculosis. Date last updated: October 2016. Date last accessed: April 2018. https://apps.who.int/iris/bitstream/handle/10665/250125/ 9789241549639-eng.pdf;jsessionid=605E8FC225283D2EA72288.

12 Walker TM, Kohl TA, Omar SV, et al. Whole-genome sequencing for prediction of Mycobacterium tuberculosis drug susceptibility and resistance: a retrospective cohort study. Lancet Infect Dis 2015; 15: 1193-1202.

13 Maguire H, Brailsford S, Carless J, et al. Large outbreak of isoniazid-monoresistant tuberculosis in London, 1995 to 2006: case-control study and recommendations. Euro Surveill 2011; 16: 19830.

14 Smith CM, Trienekens SC, Anderson C, et al. Twenty years and counting: epidemiology of an outbreak of isoniazid-resistant tuberculosis in England and Wales, 1995 to 2014. Euro Surveill 2017; 22: 30467.

15 Stagg HR, Abubakar I, Brown J, et al. Towards better guidance on caseload thresholds to promote positive tuberculosis treatment outcomes: a cohort study. BMC Med 2016; 14: 52.

16 Potter JL, Capstick T, Ricketts WM, et al. Streptomycin. TB Drug Monographs. www.tbdrugmonographs.co.uk/ streptomycin.html Date last accessed: July 2019. Date last updated: August 2018.

17 Fregonese F, Ahuja SD, Akkerman OW, et al. Comparison of different treatments for isoniazid-resistant tuberculosis: an individual patient data meta-analysis. Lancet Respir Med 2018; 6: 265-275.

18 Gegia M, Winters N, Benedetti A, et al. Treatment of isoniazid-resistant tuberculosis with first-line drugs: a systematic review and meta-analysis. Lancet Infect Dis 2017; 17: 223-234.

19 Menzies D, Benedetti A, Paydar A, et al. Standardized treatment of active tuberculosis in patients with previous treatment and/or with mono-resistance to isoniazid: a systematic review and meta-analysis. PLoS Med 2009; 6: e1000150. 
20 Stagg HR, Harris RJ, Hatherell HA, et al. What are the most efficacious treatment regimens for isoniazid-resistant tuberculosis? A systematic review and network meta-analysis. Thorax 2016; 71: 940-949.

21 Gillespie SH, Crook AM, McHugh TD, et al. Four-month moxifloxacin-based regimens for drug-sensitive tuberculosis. N Engl J Med 2014; 371: 1577-1587.

22 Jindani A, Harrison TS, Nunn AJ, et al. High-dose rifapentine with moxifloxacin for pulmonary tuberculosis. N Engl J Med 2014; 371: 1599-1608.

23 Kang YA, Shim TS, Koh WJ, et al. Choice between levofloxacin and moxifloxacin and multidrug-resistant tuberculosis treatment outcomes. Ann Am Thorac Soc 2016; 13: 364-370.

24 Maitre T, Petitjean G, Chauffour A, et al. Are moxifloxacin and levofloxacin equally effective to treat XDR tuberculosis? J Antimicrob Chemother 2017; 72: 2326-2333.

25 Sarathy J, Blanc L, Alvarez-Cabrera N, et al. Fluoroquinolone efficacy against tuberculosis is driven by penetration into lesions and activity against resident bacterial populations. Antimicrob Agents Chemother 2019; 63: e02516-18.

26 Al-Shaer $\mathrm{MH}$, Alghamdi WA, Alsultan A, et al. Fluoroquinolones in drug-resistant tuberculosis: culture conversion and pharmacokinetic/pharmacodynamic target attainment to guide dose selection. Antimicrob Agents Chemother 2019; 63: e00279-19.

27 Deshpande D, Pasipanodya JG, Mpagama SG, et al. Levofloxacin pharmacokinetics/pharmacodynamics, dosing, susceptibility breakpoints, and artificial intelligence in the treatment of multidrug-resistant tuberculosis. Clin Infect Dis 2018; 67: S293-S302.

28 Thai PVK, Ha DTM, Hanh NT, et al. Bacterial risk factors for treatment failure and relapse among patients with isoniazid resistant tuberculosis. BMC Infect Dis 2018; 18: 112.

29 Marrakchi H, Ducasse S, Labesse G, et al. MabA (FabG1), a Mycobacterium tuberculosis protein involved in the long-chain fatty acid elongation system FAS-II. Microbiology 2002; 148: 951-960.

30 Seifert M, Catanzaro D, Catanzaro A, et al. Genetic mutations associated with isoniazid resistance in Mycobacterium tuberculosis: a systematic review. PLoS One 2015; 10: e0119628.

31 Zellweger JP. Treatment of tuberculosis. Expert Rev Respir Med 2007; 1: 85-97.

32 Xie YL, Chakravorty S, Armstrong DT, et al. Evaluation of a rapid molecular drug-susceptibility test for tuberculosis. N Engl J Med 2017; 377: 1043-1054. 